\title{
The hospitalized preterm newborn: The significance of parents' participation in the Neonatal Unit
}

\author{
Josefina Gallegos-Martínez ${ }^{1}$ \\ Jaime Reyes-Hernández ${ }^{1}$ \\ Carmen Gracinda Silvan Scochi ${ }^{2}$
}

Objective: to identify and analyze the significance of participation for parents in a Neonatal Unit (NU) of a maternity hospital in San Luis Potosí, Mexico. Method: a qualitative study with 20 parents of both sexes, between 18 and 39 years of age, with a preterm hospitalized child. A content analysis using a thematic approach was used for information processing. Results: the significance of: a) the NICU as a place of suffering and waiting, b) dealing with the child's admittance, c) being excluded from the hospitalized child's care and d) being aware of inadequacies in the child's care. Conclusion: parents deal with their suffering and experience barriers that impede their participation; they respond to the medical hierarchy that limits participation, even when it goes against their natural role as parents. The caregiving philosophy needs to be transformed so that parents are considered people with the right to participate and to be competent in caring for their child.

Descriptors: Neonatal Nursing; Newborn; Parents.

\footnotetext{
${ }^{1}$ PhD, Professor, Facultad de Enfermería, Universidad Autónoma de San Luis Potosí, San Luis Potosí, México.

2 PhD, Full Professor, Escola de Enfermagem de Ribeirão Preto, Universidade de São Paulo, WHO Collaborating Centre for Nursing Research Development, Ribeirão Preto, SP, Brazil.
}

Corresponding Author: Josefina Gallegos-Martínez Calle Moras, 557

Fracc. Lomas Del Camino

78146, San Luis Potosí, México

E-mail: joga2002@hotmail.com
Copyright (c) 2013 Revista Latino-Americana de Enfermagem This is an Open Access article distributed under the terms of the Creative Commons Attribution Non-Commercial License (CC BY-NC).

This license lets others distribute, remix, tweak, and build upon your work non-commercially, and although their new works must also acknowledge you and be non-commercial, they don't have to license their derivative works on the same terms. 


\section{Introduction}

Because of their health situation, preterm newborns require special care in the Neonatal Unit (NU) with periods of hospitalization that may last for days and even months. The consequence of this hospitalization can be the disruption of the parent-child relationship, which ultimately affects the child's emotional and physical development(1). It is known that parents' participation in the care of their premature child in the NU stimulates the children's development and prepares them for early discharge ${ }^{(2)}$. However, parent participation should be developed under certain premises, such as unrestricted visits, and it should be based on relationships of equality with the family, cooperation and collaboration, and changing the values and attitudes of professionals and parents. Nursing personnel should adopt a consultative role when parents are involved in the care of their child(3-5).

Parental participation has been a principle of preterm and infant care in various countries, including the United Kingdom, United States of America (US) and Brazil. Parental participation was initiated in the US in the 1950 s with joint stays (rooming-in), in the 70s in Mexico through the participating mother program and in Colombia with the kangaroo method. In the 1980s, individualized care that centered specifically on the development of preterm infants emerged in Boston, Massachusetts in the US. Additionally, the view of the mother as the sole caretaker has shifted, and fathers are now being considered an integral part of their children's care ${ }^{(1,3,6)}$.

In San Luis Potosí, Mexico, participation is not based on any law it is not systematized, is focused on the mother, is partial and is limited to one or two hours a day. Permission to enter the NU depends greatly on the preterm child's clinical situation and on the mother's milk production. In some care units, parental participation may consist of maternal care, such as feeding, hygiene, early stimulation and receiving some instructions for hospitable discharge during the predischarge period. All of this occurs without a defined program. The father, meanwhile, can only enter the NU to make observational visits or receive medical information (7). Additionally, in Mexico, very few publications on parental participation exist. Therefore, the present study is the first to show the NU experience from the father's perspective.

For this reason, the present study aims to identify and analyze the significance that parents' participation in the care of their preterm child has on the parents of children in the NU of a second-level hospital in San Luis Potosí, Mexico.

\section{Methods}

A qualitative descriptive study was conducted that would describe and understand the experience and participation of parents with a preterm child admitted to the NU. This type of descriptive study allows a deeper understanding of the meanings of actions and human relationships that are not perceptible with statistics ${ }^{(8)}$.

The study setting was a second-level care public maternity hospital with regional coverage in San Luis Potosí, Mexico. The hospital has 3240 births and approximately $600 \mathrm{NU}$ admissions yearly. The mission of this hospital is caregiving, teaching and research. The team of health professionals is composed of pediatricians, a neonatologist, nurses, a psychologist and social workers.

The participants were mothers (9) and parents (11) between 18 and 39 years of age, with a preterm child hospitalized in the $\mathrm{NU}$, who agreed to participate in the study. Confidentiality of patient identities was maintained with corresponding acronyms. Thus, (FF121CC), corresponds to the classification of Father of Family 1, 21 years old with a preterm child hospitalized in the critical care unit of the NU. The parents belonged to nuclear (70\%), one-parent (25\%) and expanded (5\%) families. The main maternal occupation was homemaker $(89 \%)$, and the main paternal occupations were laborers, workmen, bricklayers (81.8\%) and unemployed (18.2\%). Grade school was completed in less than $35 \%$ of the cases.

Parental recollections were recorded using a semistructured interview with an average duration of 45 minutes. The interviews were taped and subsequently integrally transcribed for analysis. The interviews included identification data and the leading question: What is it like to be the father/mother of a preterm baby hospitalized in the NU, and what is your role in the baby's care? While telling their stories, the participants reflected freely. To better clarify the responses that were sought, complementary questions were utilized: How does your child's hospitalization in the NU affect your family? What would you like to know or have explained about your child's hospitalization in the NU? What are the difficulties or obstacles that you have had with your child's hospitalization in the NU? How do you propose to overcome them? 
The analytical method was based on content analysis, using a thematic approach with a cut-off rule that qualitatively studies the motivations, opinions, attitudes, values, beliefs and tendencies presented in speech. Operationally, three steps were followed: a preanalysis to establish a complete understanding of the material in an integral way; an examination of the material that carefully considered the content to understand the relevant structures; and a subsequent exploration of the material. This exploration was conducted by repeatedly reading until the primary themes had been obtained and then rereading to obtain the overall sense. Finally, the data were processed and interpreted to discover the meaning of the regularities, thus revealing of the underlying content, themes or meanings and the articulations between the data and the subject under investigation ${ }^{(9)}$. The referent theory used for interpretation was the conceptual and theoretical construction of parental participation in care and the experiences of parents with a hospitalized preterm child using contributions published by investigators on the topic.

Ethical and legal considerations for the study were based on the Mexico General Health Law, Articles 3, 100,101 , relating to research involving humans ${ }^{(8-10)}$. Approval was obtained from the study's institution. The participants who were interviewed signed the terms of informed consent that stated that they could withdraw from the study freely and voluntarily without their care being affected.

\section{Results and Discussion}

The parents spoke about their experiences in the $\mathrm{NU}$, which allowed us to understand the significance of the following themes:

- The NU as a place of suffering and waiting.

- Dealing with the child's hospitalization.

- Being excluded from the care of the hospitalized child.

- Becoming aware of lacking competence to care for the sick child.

The significance of these themes was examined to gain a better understanding of them.

\section{The NU as a place of suffering and waiting.}

Parents considered the NU place of suffering; the child's hospitalization requires that he or she will remain in an inaccessible place where the parents cannot have contact with him or her. As one participant expressed: [...] After labor, I started to bathe. I experienced a very large emptiness and an immense desire to cry because I said, oh my belly! [...] I felt an emptiness when I arrived at the house and wanted to have contact with her (the daughter), we (the father and mother) saw her in the incubator, but through the window [...] (MF236CC). The parents found themselves out of place in the NU and were not familiar with hospital dynamics. Contact with their child depended on the views of the health personnel, who did not let them enter due because of their child's critical condition(11). In addition to this restriction, they had to adapt to the strange environment and specialized language and face problems they did not understand. It is known that parents in this situation can develop an acute stress disorder associated with the alteration in the parental role that takes away their ability to help their child, protect him or her from pain and care for him or her or even leave them with another caretaker. Parents also face the difficulties of taking care of other children, which also limits their participation in caring for the hospitalized child: [...] (To come) [...] (I am supposed to) leave my children in the care of others, and right now my (other) girl is sick and (I am supposed) to risk that to come here [...] (MF422CI). In a qualitative study in Mexico, the NU heath team recognized that the responsibility of caring for other children is an obstacle to parental participation in the NU and, additionally, having a child in the NU leads to a suspension of normality of family dynamics ${ }^{(12-13)}$.

The parents' anguish was also related to the child's clinical condition, treatment and medical technology because they associated it with the child's suffering and risk of death: [...] It was very shocking to see my daughter with the probe, with the pump (nebulizer), in a very bad condition, very injured [...] I felt a lot of anguish, impotence; the first thing that came to my mind was to say, 'Why her, so tiny, so defenseless, not able to say'; it hurts me (the mother cries) [...] (MF519CC). Painful feelings, feelings of anguish and sadness or other feelings, such as guilt, emerge or are reinforced [...] oh! I felt so awful, God, when I entered! No! And I saw him in the incubator, and with his IV like that, I wanted to be in his place, him so defenseless there, I felt guilty going back to work the eight months because I was working and leaving the girl in the care of someone else and I felt guilty because through my own fault, (the child) was ill [...] (MF422CI). The parents of hospitalized newborns go through different emotional states throughout hospitalization, such as guilt that deteriorates their self-esteem and depression associated with the constant fear of death. Rage appears when the parent cannot modify the baby's condition. Parents become enraged because they cannot overcome the situation; they become aggressive and hostile toward the health team because they have 
removed the parents' right to care for their baby, which causes feelings of lower worth and estrangement from their child(12-14).

The NU requires a long wait before discharge while the child's development is monitored; meanwhile, the parents routinely analyze the child's clinical changes, including respiratory function and weight gain. At the same time, parents suffer the uncertainty of death, comparing their child with other newborns in similar situations who are already deceased: [...] He is going on four months here... what more could one want than for the child to be well and all, to have him at home like the others. (MF619CC) We are following his improved respiration, and we see that he is breathing a lot better compared to the first few days [...] (MF1636CC) I see him full of apparatuses. Who knows, really, but with time we will see progress [...] all of the children who were born like him have died. I say. 'Oh God, why mine, why so much time?' and one says why this, why that if this one already died and another also, why was he born so tiny, weighing 800 grams and now already weighs 1.630 kilograms? And then I see a similar boy who, thank God, has already passed through this, right? But who knows? We just have to wait because yes, yes its difficult to be here [...] (MF619CC).

In experimental and qualitative studies with parents of preterm infants hospitalized in the NU, findings show feelings related to suffering, stress responses to the situation and the need for information and the support of health personnel, given preventive controlled interventions reduce this stress significantly. According to research-based evidence, the American Association of Pediatrics recommends parental participation because it produces multiple benefits, such as reducing parents' stress, improving the child's clinical recovery and reducing the child's pain. These benefits have led to a consideration of whether the NU's organization should favor parental participation(15-17).

\section{Dealing with the child's hospitalization}

Parents of children in the NU experience suffering caused by separation from their child. However, they are also concerned about family support because many single mothers do not have the father's support. Despite the lack of support from the father, some single mothers feel supported by their family during this difficult time: Everybody (family) gives us a hand and they all support us. (PF2023CC). In the case of single-parent families in which only the mother cares for the child, the mothers experience the hospitalization without family support: [...] I got pregnant and therefore, I am a single mother, and my parents do not support me at all. I alone am with her (the daughter) (MF920CI). And at the same time, the mothers support their child: So I speak sweetly (to the child) to encourage the child, although the dad is not here, and so my family has come to see him. (MF1723CC). The father is present in some cases: All my life, I have been on the street, and I almost never lived with my family. Until now, we have been living together, and now that my two children (twins) arrived, just being in the house with my wife, taking care of her and knowing that she needs me and there I am with her, and that's why my life is transformed. (FF721CC). Family support becomes a source of strength for dealing with the situation that the parents face, as also described in other similar studies ${ }^{(18)}$.

The financial question of paying for hospital care increases worry and stress because many parents are adolescents and youths who have scarce family income or means to settle the bills: I hope that she (daughter) is able to leave soon, because the truth is that maybe they will charge us a lot [...] Right now, while I am here, I am trying to set up government aid, and the truth of how much time has already been spent here and if they charge me [...] as he was premature, if it covers him [...] I just lost my job, because I came right away, and then I notified my bosses and they knew that I would be coming here because my wife was getting better, I was coming here to be with her and with the girl to take care of what she needed, and I said good-bye to her, and at that time I went to see if I could find another job [...] This week, I obtained 1000 pesos to come. I bought the medicine, and already there go 10,000 pesos [...] (FF121CC).

Mexican families invest an average of $50 \%$ of the family economic income in health care problems, which is a factor of social impoverishment. Presently, support for disastrous expenses for a population that does not have social security is available through government aid. This aid includes payment for the neonate's hospitalization, although it does not cover everything for low-income families. There are other types of expenses, such as the trips to the municipal headquarters during hospitalization or the medical equipment needed to continue care for the baby in the home at the parents own expense ${ }^{(6,18-19)}$

\section{Being excluded from the care of the hospitalized child}

Fathers experience a gender distinction on the part of the NU health team. They perceive that the NU team views them as having little importance, sees only the mother as the central contributor to the sick child's care, and considers the father a vehicle of infections: [...] only from the outside [...] they do not let me (enter the NU) [...] it doesn't feel the same seeing her from a 
distance compared to up close [...] I wanted to hold her, but maybe its better like this, so she will get better soon, only her mom enters, maybe I don't have to be there because she is more important for taking care of her because she is the mom [...] maybe it is because they put them in the incubators so they do not let us enter maybe because sometimes you can bring a cold or I don't know; it complicates things [...] (FF1018CC). The parents consider that the most important thing for them is that the mother communicates with the child, that it increases her feelings of confidence and closeness with the child: [...] I imagine it as if I was watching her, and it calms me down more [...] My wife tells me: "At times, she cries. Her mouth moves; if she is hungry she searches and it moves" [...] So it is a long visit, and I do not dwell on the matter (that they do not let the father in). My wife later comes to feed him. I see her, and she talks to me [...] (FF121CC).

The father gains importance in communicating with the health team about the state of the child, and it is he who transmits information to the mother: [...] I want them to tell me how it is: how he is doing, how he is reacting. At times it would be nice (to know) if he has improved and how to deal with it, since sometimes my husband comes (to know) what news he (the doctor) left, how he (the child) is coming along in his illness [...] (MF1533CC) [...] We ask (the doctor), and they give us a diagnosis of how the child is coming along, to know about the injection medicines and all that [...] what any father wants to know is whether they give a good diagnosis and when we can take him home [...] (FF1939CC). The reality of the parent participation in NUs in Latin American countries is shown in studies. In one descriptive study of nine NUs in Mexico, the fathers only perform the role of observers and receivers of information(7). In Brazil, a qualitative study found that fathers are interested in actively participating in the lives of their children, but when they care for their child in the hospital, they feel excluded, not considered by the nursing staff and simultaneously admired and negatively viewed by the mothers of other children for being a man ${ }^{(20)}$.

The mother's participation is also limited, although in a different way from the father's. The health team allows mothers to enter the NU, particularly when they are capable of producing milk, to nurse the baby or to deliver milk: [...] They always let me to come see her from outside, or sometimes they tell me to come so I can see her [...] I still don't (participate) because I still don't produce milk [...] (MF519CC) From a distance, because I couldn't touch her; well, far away on one side of the incubator because before I had to see her through glass; that is, not even near the incubator [...] Why do they do this? I don't agree, it is offensive [...] I saw it as an obstacle [...] I am participating by bringing my milk [...]
(MF1636CC). The mother is seen principally as a provider of food; that is the key to entering the NU, and this view of mothers is similar in other hospitals in San Luis Potosi(7).

\section{Being aware of inadequacies in the care of the sick child}

The experience of observing the hospitalized child as the subject of technological care, undergoing incomprehensible pharmacological treatments in a complex environment such as the NU, combined with such circumstances as being the parents' first child or the parents being particularly young, makes parents feel unable to provide the basic care that the child should receive: [...] Well, I don't know anything about babies right? Because I have been taking care of him, but since he is so tiny, this one [...] So yes, I would like them to tell me how to bathe him, what to feed him, if what I already did and is okay, or when he cries or when does something else (what to do), because I have already held him, but since they are so tiny [...] (MF319CI) Be careful giving her the bottle, be careful with her (the daughter) or maybe later she will get sick [...] (FF121CC) More than anything, for the care of the babies, what kind of care they will need, in changing them, dressing them, bathing them, for everything [...] (FF721CC) Yes, you must keep this in mind because you must know what to do with him, because he is small and I am going to spend so much on being here and the cares that he needs, since they told me about everything (caregiving); he is the first one. Yes, I enter (the NU); 5 minutes is very little [...] he also has oxygen here in his little head or in his hand, and I don't do much more than hold him [...] (MF619CC). The participation of the parents, above all of the mother through her social role of main caretaker, has been discussed in the literature. A qualitative study found that parents experience a process that goes from being far removed from participating in the care of the child; they go from a passive role to an active one with a focus that is transformed from being centered on what happens in the $\mathrm{NU}$, to being centered on the care of the child, for which they initially lack caretaking competencies. This may lead the parents to consider the nurses, who are gifted with very specialized technical abilities, as rivals in the care of the child. Therefore, the mothers, in addition to feeling that they have no technical competence in the care of their own child, must tell the nursing team about their lack of competence to receive the necessary orientation. To complement this, the team should recognize that both parents should be qualified in basic caretaking and some specific caretaking tasks for their sick child hospitalized in the NU if they are to be 
able to carry out these tasks in the home ${ }^{(6)}$.

On the other hand, the parents also discussed how they could improve participation: I said to put up a shelter, even if its a small one, because there are many people that are not from here, they come from a distance to be here with their child. Sometimes they do not bring anything to eat, nothing, and here they always are. This is what I would want or what I would need [...] (MF519CC). Such improvements can be achieved if they are supported to satisfy the parents' needs, if caretaker education programs are developed for parents both in the hospital but in the home also so that they contribute in some way to humanized care $\mathrm{e}^{(6,11,18)}$.

\section{Final considerations}

Parental participation in the NU is extremely rare, with little conscious recognition on the part of the parents of these children. The rarity of this experience leads to suffering, which is significant in the process of personalized caretaking. However, these same parents perceive the limits placed on their participation as a normal part of what happens in the NU under the hospital hierarchy. They adapt to these rules in an anti-empowering way and do not consider that they should have opportunities for emancipation and active participation in the care and decisions concerning their hospitalized child.

The anticipated sorrow that the parents experience affects their subjectivity however, even this sadness does not move them to look for solutions for the care of their child. All of these experiences seem to be permeated by a lack of knowledge and ability to provide adequate care. This leads us to consider the need for a transformation of traditional neonatal caretaking based on the separation and appropriation of the biological body of the sick newborn and unilateral decision-making in a way that is detrimental to the parents' natural role, moving instead toward systematized, active parental participation.

The possibility of a paradigm change from a focus on the biological aspect of the child toward a humanized care that includes the family is still in its early stages. The redefinition of health professionals' roles would require adopting a vision that integrates human resources training and health. Professional duties would center on the humanization of caretaking in an organized and systematic way that focuses on subject participation and considers concepts such as quality of life, empowerment, emancipation, citizenship, human rights, ethics and absence of risk in neonatal caretaking.

\section{Constraints and recommendations}

Due to the importance of the theme of parental competencies in caretaking and the approach toward caretaking, which was covered in less depth, we recommended that subsequent studies in this field should take caretaking as their main focus, given that related studies show that it is an urgent need. Future investigations could incorporate educational interventions directed toward parents and should educate the heath team with respect to the educational needs of this population. Such interventions would be based on a philosophy of care centered on the family and individualized care centered on the development of the newborn.

\section{References}

1. Talmi A, Harmon RJ. Relationships between preterm infants and their parents: disruption and development. Rev Zero to Three. 2003;103-20.

2.American Academy of Pediatrics. Hospital discharge of the High-Risk Neonate. New AAP Policy Statement. Pediatrics. 2008;122(5):1119-26.

3. Coyne IT, Cowleyb S. Challenging the philosophy of partnership with parents: A grounded theory study. Int J Nurs Stud. 2007;44:893-904.

4.Lima RGA, Rocha SMM, Scochi CGS. care to hospitalized children: reflections about parents'. Rev. Latino-Am. Enfermagem. 1999;7(2):33-9.

5. Fegran L, Solveig FM, Helseth S. Development of parent-nurse relationships in neonatal intensive care units - from closeness to detachment. J Adv Nurs. 2008;64(4):363-71.

6. Gallegos-Martinez J, Monti-Fonseca LM, Silvan-Scochi CG. The participation of parents in the care of premature children in a neonatal unit: meanings attributed by the health team. Rev. Latino-Am. Enfermagem. 2007;15(2):239-46.

7. Gallegos-Martínez J, Reyes-Hernández J, SilvanScochi CG. La Unidad Neonatal y la participación de los padres en el cuidado del prematuro. Rev Perinat Reprod Hum. abr-jun 2010;24(2):98-108.

8. Minayo MC. Pesquisa social: teoria, método e creatividade. 19.ed. Petrópolis: Vozes; 2001. 80 p.

9. Bardin L. Analise de conteúdo. Lisboa Edições; 1977. 10. Ley General de Salud (MX). Diario oficial de la Federación; 1997.

11. Wigert $H$, Hellström AL, Berg M. Conditions for 
parents' participation in the care of their child in neonatal intensive care - a field study. BMC Pediatrics. 2008;8:3 12. Heermann LA, Wilson ME, Wilhelm PA. Mothers in the NICU: Outsider to Father. Pediatric Nurs. 2005;31(3):176-200.

13 Kendall-Tackett K. Traumatic Stress symptoms in parents of premature infants. Trauma Psychol Newsletter. 2009:16-8.

14. Argote L, Montes M. La Donación hace la diferencia en el cuidado de padres y niños prematuros. Fam Saúde Desenv. 2002;4(1):7-15.

15. Jotzo M, Poets CF. Helping parents cope with the trauma of premature birth: An evaluation of a traumapreventive psychological intervention. Pediatrics. 2005;115(4):915-9.

16. Fraga ITG, Pedro ENR. Sentimentos das mães de recém-nascidos prematuros: implicações para a enfermagem. Rev Gaúcha Enfem. 2004;25(1):89-97.

17. American Academy of Pediatrics. Committee on Hospital Care Policy Sttatement. FamilyCentered Care and the pediatricians role. Pediatrics. 2003;112(3):691-7.

18. Siqueira $M B C$, Dias MAB. A percepção materna sobre vivência e aprendizado de cuidado de um bebê prematuro. Epidemiol Serv Saúde. 2011;20(1):27-36.

19. González-Rubio AB. Fondo de Protección contra Gastos Catastróficos. México-Secretaría de Salud. [Internet]; [acesso 13 jun 2012]; Disponivel em: http://www.seguro- popular.salud.gob.mx/index. php?option=com_content $\&$ view $=$ article $\&$ id $=84 \& \% 20$ Itemid $=115$.

20. Moura EV, Ribeiro NR. El padre en el contexto hospitalario infantil. Rev Gaúcha Enferm. $2004 ; 25(3): 386-95$. 